\title{
Thermoelectric properties of minerals with the mawsonite structure
}

Article

Accepted Version

Siloi, I., Gopal, P., Curtarolo, S., Nardelli, M. B., Vaqueiro, P. and Fornari, M. (2019) Thermoelectric properties of minerals with the mawsonite structure. ACS Applied Energy Materials, 2019 (2). pp. 8068-8078. ISSN 2574-0962 doi:

https://doi.org/10.1021/acsaem.9b01564 Available at https://centaur.reading.ac.uk/87111/

It is advisable to refer to the publisher's version if you intend to cite from the work. See Guidance on citing.

To link to this article DOI: http://dx.doi.org/10.1021/acsaem.9b01564

Publisher: ACS Publications

All outputs in CentAUR are protected by Intellectual Property Rights law, including copyright law. Copyright and IPR is retained by the creators or other copyright holders. Terms and conditions for use of this material are defined in the End User Agreement.

\section{www.reading.ac.uk/centaur}

\section{CentAUR}

Central Archive at the University of Reading 
Reading's research outputs online 


\section{ACS APPLIED ENERGY MATERIALS}

\section{Article}

\section{Thermoelectric Properties of Minerals with the Mawsonite Structure}

Ilaria Siloi, Priya Gopal, Stefano Curtarolo, Marco Buongiorno Nardelli, Paz Vaqueiro, and Marco Fornari

ACS Appl. Energy Mater., Just Accepted Manuscript • DOI: 10.1021/acsaem.9b01564 • Publication Date (Web): 31 Oct 2019

Downloaded from pubs.acs.org on November 1, 2019

\section{Just Accepted}

"Just Accepted" manuscripts have been peer-reviewed and accepted for publication. They are posted online prior to technical editing, formatting for publication and author proofing. The American Chemical Society provides "Just Accepted" as a service to the research community to expedite the dissemination of scientific material as soon as possible after acceptance. "Just Accepted" manuscripts appear in full in PDF format accompanied by an HTML abstract. "Just Accepted" manuscripts have been fully peer reviewed, but should not be considered the official version of record. They are citable by the Digital Object Identifier (DOI®). "Just Accepted" is an optional service offered to authors. Therefore, the "Just Accepted" Web site may not include all articles that will be published in the journal. After a manuscript is technically edited and formatted, it will be removed from the "Just Accepted" Web site and published as an ASAP article. Note that technical editing may introduce minor changes to the manuscript text and/or graphics which could affect content, and all legal disclaimers and ethical guidelines that apply to the journal pertain. ACS cannot be held responsible for errors or consequences arising from the use of information contained in these "Just Accepted" manuscripts. 


\title{
Thermoelectric Properties of Minerals with the
}

\section{Mawsonite Structure}

\author{
Ilaria Siloi, ${ }^{*},, \|$ Priya Gopal, ${ }^{\dagger}$ Stefano Curtarolo, ${ }^{\ddagger}, \perp$ Marco Buongiorno \\ Nardelli, ${ }^{\dagger, \perp}$ Paz Vaqueiro, " and Marco Fornari*, ${ }^{*, \perp}$ \\ Department of Physics, University of North Texas, Denton, TX 76203, USA, Department \\ of Mechanical Engineering and Materials Science, Duke University, Durham, NC 27708, \\ USA, Department of Chemistry, University of Reading, Whiteknights, Reading, RG6 6AD, \\ UK, and Department of Physics and Science of Advanced Materials Program, Central \\ Michigan University, Mt. Pleasant, MI 48858, USA \\ E-mail: siloi@usc.edu; marco.fornari@cmich.edu
}

\begin{abstract}
Synthetic copper sulfides have emerged as promising non-toxic and low-cost materials for thermoelectric power generation in low-grade waste heat recovery systems. Similarly to tetrahedrite and colusite, mawsonite $\mathrm{Cu}_{6} \mathrm{Fe}_{2} \mathrm{SnS}_{8}$ exhibits a modified corner sharing $\mathrm{Cu}-\mathrm{S}$ tetrahedral network which usually leads to p-type character and low thermal conductivity. In order to explore the applicative potential of mawsonite, we studied
\end{abstract}

\footnotetext{
*To whom correspondence should be addressed

${ }^{\dagger}$ Department of Physics, University of North Texas, Denton, TX 76203, USA

${ }^{\ddagger}$ Department of Mechanical Engineering and Materials Science, Duke University, Durham, NC 27708, USA

『Department of Chemistry, University of Reading, Whiteknights, Reading, RG6 6AD, UK

$\S$ Department of Physics and Science of Advanced Materials Program, Central Michigan University, Mt. Pleasant, MI 48858, USA

"Present address: Department of Physics and Astronomy, University of Southern California, Los Angeles, CA 90089, USA

${ }^{\perp}$ Center for Autonomous Materials Design, Duke University, Durham, NC 27708, USA 
the band structure, the phonon dispersions, the electronic and transport coefficients as well as the effect of isovalent substitutions of Fe, Sn and S. The combined analysis of electronic and vibrational properties highlights the role of the weakly bonded copper component in achieving a very low thermal conductivity. We also demonstrate that the $\mathrm{Cu}-\mathrm{S}$ bond builds a 2D conductive network where the contribution from other elements is negligible. Magnetic calculations point to an anti-ferromagnetic ground state substantially affected by the covalency of the bonds with the conductive plane. The chemical substitution of $\mathrm{Fe}$ with $\mathrm{Ni}$ leads to non-magnetic metals whereas $\mathrm{Cu}_{6} \mathrm{Fe}_{2} \mathrm{SnSe}_{8}$, $\mathrm{Cu}_{6} \mathrm{Fe}_{2} \mathrm{PbS}_{8}$, and $\mathrm{Cu}_{6} \mathrm{Fe}_{2} \mathrm{GeX}_{8}$ with $\mathrm{X}=\mathrm{S}$, Se, and Te are semiconductors.

\section{Introduction}

Thermoelectric (TE) devices provide waste-heat recovery solutions that are robust, environmentally friendly, and maintenance-free. Available estimates ${ }^{1,2}$ indicate that thermal losses exceed $50 \%$ of the total energy production of the United States, underlining that even marginally efficient conversion may have an enormous impact on the economies of industrialized countries. Although there have been significant advances in TE performance for power generation at high temperatures, there is a dearth of low-cost and sustainable materials for the recovery of industrial low-grade waste heat $(\mathrm{T} \leq 600 \mathrm{~K})$ which according to recent estimates represents about $60 \%$ of industrial emissions. ${ }^{3}$ To the best of our knowledge, the most promising materials in this temperature window are $\beta-\mathrm{ZnSb}_{3}{ }^{4}(\mathrm{ZT}=1.3 @ 670 \mathrm{~K})$, $\mathrm{AgPb}_{18} \mathrm{SbTe}_{20}{ }^{5}(\mathrm{ZT}=2.2 @ 800 \mathrm{~K}), \mathrm{Mg}_{2} \mathrm{Si}_{1-\mathrm{x}} \mathrm{Sn}_{\mathrm{x}}{ }^{6}(\mathrm{ZT}=1.3 @ 700 \mathrm{~K}), \mathrm{Na}_{1}-\mathrm{xPb}_{\mathrm{m}} \mathrm{SbTe}_{\mathrm{m}+2}{ }^{7}$ $(\mathrm{ZT}=1.7 @ 650 \mathrm{~K}), \mathrm{PbTeSrTe}^{8}(\mathrm{ZT}=2.5 @ 923 \mathrm{~K}), \mathrm{PbTe}-\operatorname{AgTeLa}^{9}(\mathrm{ZT}=1.6 @ 775 \mathrm{~K})$.

Traditional TE devices are engineered using doped p- and n- legs to form a thermocouple in which heat current flows in parallel whereas charge flows in series. Ideally, in each leg, the

only carriers available are holes or electrons: the combination of low thermal conductivity $(\kappa)$ and large power factor $\left(S^{2} \sigma\right.$, where $S$ is the Seebeck coefficient and $\sigma$ is the electrical conductivity) is essential for the generation of large voltage when a temperature gradient is 
applied through the device. The energy conversion efficiency of a TE material is expressed in terms of the figure of merit $Z T=S^{2} \sigma T / \kappa$ - where $T$ is the temperature at which the device operates.

Increasing the efficiency of the thermoelectric energy conversion is a difficult task which requires the optimization of intertwined electrical and thermal degrees of freedom. The main strategies used to target improved TE performance have been based on the joint effort of synthesis, characterization, and theoretical/computational studies. This is the case of the band-engineering, a procedure that relies on the accurate knowledge of the electronic structure to increase the effective masses and the number of carriers pockets at the Fermi energy with the goal to optimize the power factor. ${ }^{10-12}$ More recently, the manipulation of structural and bonding features that affect the electron relaxation time has also been successful. ${ }^{13}$ The optimization of lattice thermal conductivity, $\kappa_{\text {lat }}$, can be controlled by tuning the anharmonicity of the bonds ${ }^{14,15}$ or by introducing additional scattering channels for the heat carrier vibrations. This design criterion to reduce lattice thermal conductivity has been pursued by disrupting the crystalline order through the inclusion of heavy elements in the crystal structure (e.g. $\mathrm{AgPbSbTe}_{2},{ }^{5} \mathrm{Bi}_{2} \mathrm{Te}_{3},{ }^{16} \mathrm{BaLaYbCoSb}_{12},{ }^{17}$ ) or through nanostructuring. ${ }^{18}$ Unfortunately, most of the new materials turned out to include rare, expensive, or/and toxic chemical elements making them less ideal for technological deployment.

In the last decade, the need to conciliate performance with environmental and cost constraints has triggered research toward synthetic sulfide minerals, which often contain nontoxic and Earth-abundant elements. Despite the sulfur's light atomic weight and the strong ionic character, which are usually detrimental to the TE effects, sulfides emerged as good candidates for p-type TE conversion. This is the case of the superionic phase of chalcocite ${ }^{19}$ $\mathrm{Cu}_{2-\mathrm{x}} \mathrm{S}(0.5<Z T<1.7)$, where disordered $\mathrm{Cu}$ ions contribute to extremely low thermal conductivity while keeping electrical properties similar to normal semiconductors. The strong migration of $\mathrm{Cu}$ ions can be mitigated by adding other metallic species into the composition extending the exploration to ternary/quaternary compounds, like tetrahedrite ${ }^{20,21}$ 
$\mathrm{Cu}_{12-\mathrm{x}} \mathrm{M}_{\mathrm{x}} \mathrm{Sb}_{4} \mathrm{~S}_{13}$ with $\mathrm{M}=\mathrm{Mn}, \mathrm{Fe}, \mathrm{Co}, \mathrm{Ni}, \mathrm{Zn}$, colusite ${ }^{22,23} \mathrm{Cu}_{26} \mathrm{~V}_{2} \mathrm{Sn}_{6} \mathrm{~S}_{32}$, bornite ${ }^{24} \mathrm{Cu}_{5} \mathrm{FeS}_{4}$, stannoidite ${ }^{25} \mathrm{Cu}_{8} \mathrm{Fe}_{3} \mathrm{Sn}_{2} \mathrm{~S}_{12}$ and germanite ${ }^{26} \mathrm{Cu}_{26} \mathrm{Fe}_{4} \mathrm{Ge}_{4} \mathrm{~S}_{32}$, to mention a few. In particular tetrahedrite and chalcocite are among the most widespread ores on Earth. The largest values for $\mathrm{ZT}$ in sulfide minerals were reported near 0.5 at $500 \mathrm{~K}$ for pristine compositions without specific optimization (see for instance Ref. Pavan Kumar et al. ${ }^{13}$, Fig. 10) making this family of materials of great interest for low grade waste-heat recovery.

In this manuscript we present a first-principles theoretical study of the bulk properties of mawsonite $\mathrm{Cu}_{6} \mathrm{Fe}_{2} \mathrm{SnS}_{8}$, a sulfide mineral found in hydrothermal copper ores within volcanic rocks. Our results are discussed in light of a recently published experimental study ${ }^{27}$ that shows very low value for lattice thermal conductivity, below $1 \mathrm{~W} \mathrm{~m}^{-1} \mathrm{~K}^{-1}$ in the range of temperature $300<\mathrm{T}<650 \mathrm{~K}$, and the figure of merit $\mathrm{ZT}=0.43$ at $\mathrm{T}=623 \mathrm{~K}$ for the undoped structure. Besides providing some considerations on the crystal structure presented in Zhang et al. ${ }^{27}$, we analyse the band structure and the vibrational dispersion to rationalize the electronic transport properties and the magnetic properties. In particular, we identify the special role of the weakly bonded $\mathrm{Cu}$ component in lowering the frequency of optic modes. We will show that the vibrational properties in weakly bonded subsystems display marked similarities to the ones observed in liquids or amorphous solids. In this conceptual framework, the low thermal conductivity strictly depends on the coexistence of weakly bonded quasiliquid subsystems, $\mathrm{CuS}_{4}$, within a crystalline matrix. The anti-ferromagnetism induced by Fe as well as the consequence of the covalency of the $\mathrm{Cu}-\mathrm{S}$ bonds is also discussed. We also assess the effect of chemical tuning in mawsonite by replacing Fe with $\mathrm{Ni}$, S with Se and Te, and $\mathrm{Sn}$ with Ge, and $\mathrm{Pb}$. 


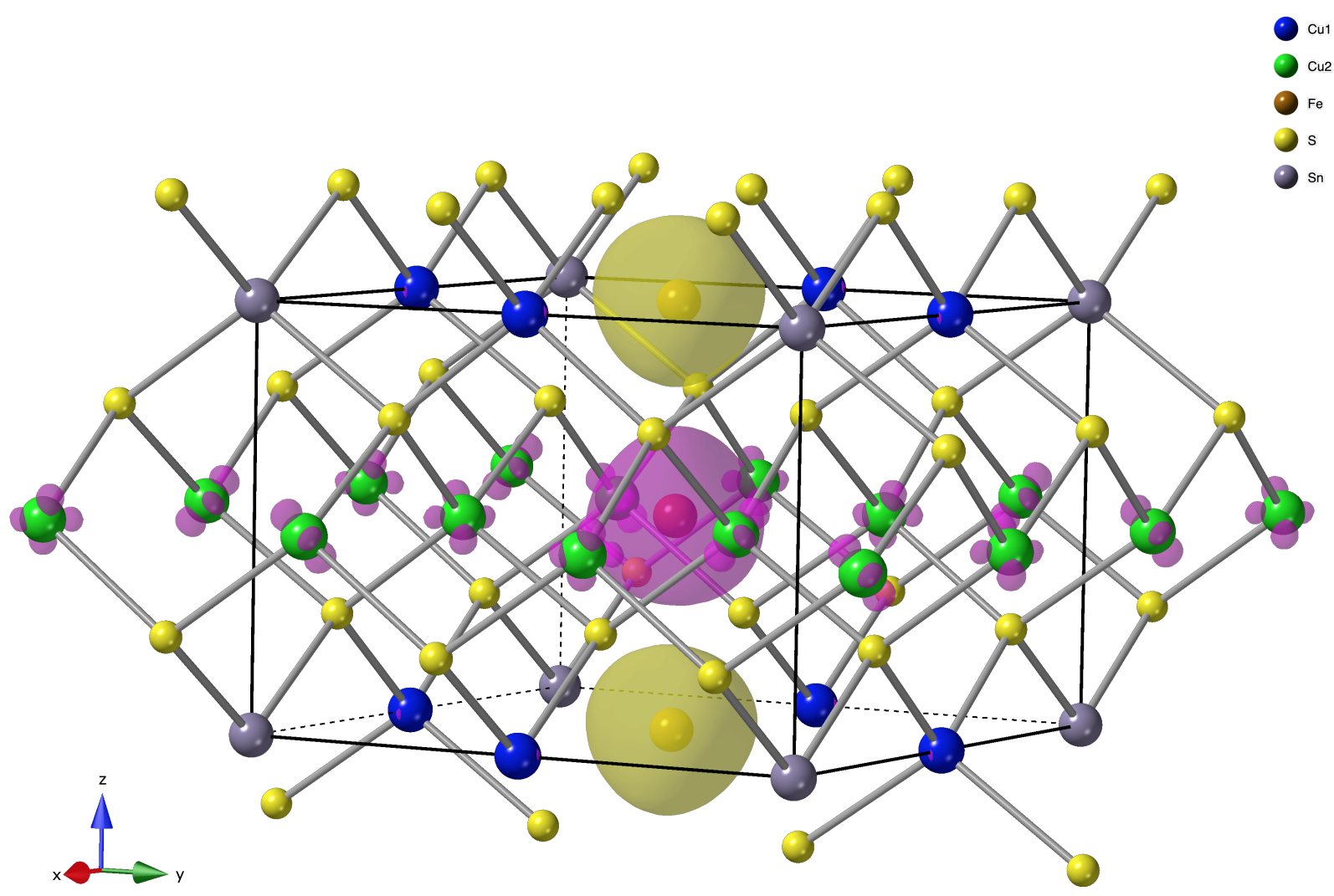

Figure 1: Unit cell of mawsonite $\mathrm{Cu}_{6} \mathrm{Fe}_{2} \mathrm{SnS}_{8}$ (space group $\mathrm{N}^{\circ} 115$ ) with $\mathrm{S}(4 \mathrm{j})$ and $\mathrm{S}(4 \mathrm{k})$ (yellow) corner-sharing tetrahedral network centered around $\mathrm{Cu}(4 \mathrm{i})$ (green) and $\mathrm{Cu}(2 \mathrm{~g})$ (blue), $\mathrm{Fe}(1 \mathrm{~b})$ and $\mathrm{Fe}(1 \mathrm{c})$ (red) and $\mathrm{Sn}(1 \mathrm{a})$ (gray) ions. Isosurface of the spin polarization positive (yellow)/negative (magenta) indicates the AFM ordering as well as a presence of an induced spin polarization on the plan shared by $\mathrm{Fe}$ and $\mathrm{Cu}(4 \mathrm{i})$. 


\section{Computational methods}

All calculations in this work are based on spin-polarized density functional theory (DFT) as implemented in Quantum Espresso ${ }^{28,29}$ integrated in the high-throughput infrastructure of AFLOW $\pi+$ PAOFLOW ${ }^{30,31}$ which allows for an automated and simplified calculation of vibrational spectra, elastic properties, dielectric functions, and transport coefficients among the others. With respect to traditional approaches for computing solids properties based on plane-wave basis set, PAOFLOW provides an effective and accurate representation of the $a b$ initio electronic structure in terms of localized basis set. Once the ab initio electronic structure is solved on a well converged plane-wave basis set, it is mapped into a tight binding (TB) model that precisely reproduces a selected number of bands of interest. The Hamiltonian for a specific material, is computed in real space using pseudo atomic orbitals from the pseudopotential of any given element as basis set. With this method one can first build and then operate with the real space ab initio TB Hamiltonian that accurately reproduces the DFT band structure. This procedure is convenient especially when the use of a very dense k-point grid is preferable, as it happens in the computation of transport properties. Further, the TB representation allows the efficient computation of two-electron integrals for the development of a local exchange functional. The ACBN0 functional approach provides a direct and self-consistent evaluation of the on-site Coulomb $U$ and exchange $J$-parameters at a low computational cost. ${ }^{32}$ Better accuracy for lattice parameters, energy band gap and phonon dispersions are obtained. ${ }^{33}$ Initially, norm-conserving (NC) PBE pseudopotentials $^{34}$ (energy cut off of $250 \mathrm{Ry}$ and $6 \times 6 \times 8$ grid) are used to determine the effective Hubbard corrections. Even though our implementation of ACBN0 uses norm-conserving pseudo-potentials, the transferability of the $U$-values has been tested on different pseudopotentials. ${ }^{35}$ The phonons calculations use ultrasoft pseudopotentials (energy-cut off of 45 Ry and $6 \times 6 \times 8$ grid) with the PBESol exchange-correlation functional ${ }^{36}$ and Hubbard U corrections reported in the caption of Figure 3. 
Transport properties - Seebeck coefficient $S$, electrical conductivity $\sigma$ and electronic contribution to thermal conductivity $\kappa_{e l}$ - are computed by solving Boltzmann transport equation within the constant relaxation time and the rigid band approximation as implemented in PAOFLOW. ${ }^{37}$ In agreement with similar compound, colusite ${ }^{23}$, we set to $10^{-15} \mathrm{~s}$ when estimating the resistivity. A very dense interpolated k-mesh of $18 \times 18 \times 24$ grid is used for accurate calculations. When discussing the electronic structure, we report transport properties across a large range of temperature whereas the chemical potential is considered constant throughout the temperature range. PAOFLOW provides the generalized transport tensors by independent calculations for spin-majority $(\uparrow)$ and spin-minority $(\downarrow)$.

$$
\mathcal{L}_{\alpha}^{s}=\frac{1}{4 \pi^{3}} \int \tau \sum_{n} \mathbf{v}_{n}^{s}(\mathbf{k}) \otimes \mathbf{v}_{n}^{s}(\mathbf{k}) \frac{\partial f_{0}}{\partial \epsilon^{s}}\left[\epsilon_{n}^{s}-\mu\right] d \mathbf{k}
$$

with $\alpha=0,1,2, s=\uparrow, \downarrow$ is the spin index, $\tau$ is the constant scattering time, $\mathbf{v}_{n}(\mathbf{k})$ is the electron velocity for the $n$-th band on each $k$-point, $f_{0}$ is the equilibrium distribution function and $\epsilon$ is the electron energy in each spin configuration $s$. Taking into account the spin polarization, the relevant transport quantities can be defined according to the two-current model $^{38}$ in which the spin-flip scattering is neglected:

$$
\begin{aligned}
& \sigma=e^{2}\left(\mathcal{L}_{0}^{\uparrow}+\mathcal{L}_{0}^{\downarrow}\right), \\
& S=-\frac{1}{T e}\left(\left[\mathcal{L}_{0}^{\uparrow}\right]^{-1} \cdot \mathcal{L}_{1}^{\uparrow}+\left[\mathcal{L}_{0}^{\downarrow}\right]^{-1} \cdot \mathcal{L}_{1}^{\downarrow}\right), \\
& \kappa_{e l}=\frac{1}{T}\left[\left(\mathcal{L}_{2}^{\uparrow}+\mathcal{L}_{2}^{\downarrow}\right)-\left(\mathcal{L}_{1}^{\uparrow} \cdot\left[\mathcal{L}_{0}^{\uparrow}\right]^{-1} \cdot \mathcal{L}_{1}^{\uparrow}+\right.\right. \\
&\left.\mathcal{L}_{1}^{\downarrow} \cdot\left[\mathcal{L}_{0}^{\downarrow}\right]^{-1} \cdot \mathcal{L}_{1}^{\downarrow}\right] .
\end{aligned}
$$

Vibrational properties have been obtained using the finite-displacement method as implemented in Quantum ESPRESSO with a supercell of $2 \times 2 \times 2$, whose volume has been relaxed first. Between each ionic step of the geometry optimization the force convergence tolerance is set to $10^{-3} \mathrm{Ry} /$ Bohr, while the energy tolerance is $10^{-4}$ Ry to ensure accuracy. In the self- 
consistent electronic calculation instead the energy threshold is set to $10^{-10} \mathrm{Ry}$. The phonon dispersion provides a starting point for the computation of the lattice thermal conductivity, as expressed in the Debye-Callaway model. ${ }^{39,40}$ Even though the integrated Grüneisen parameter is not directly correlated to thermal conductivity, ${ }^{41}$ the frequency distribution of the Grüneisen parameter resolved by modes seems to be a good qualitative descriptor for the lattice thermal conductivity. ${ }^{15,42,43}$ Within the quasiharmonic approximation we compute the mode resolved Grüneisen parameter $\gamma_{q j}$ for the wave vector $q$ and the phonon branch $j$ by taking the derivative of the dynamical matrix with respect to the volume:

$$
\gamma_{q j}=\frac{V_{e q}}{2 \omega_{q j}} \sum_{j} e_{q j} \frac{\partial D_{q}}{\partial V} e_{q j}^{*}
$$

where $D_{q}$ is the dynamical matrix for a wave-vector $q, \omega_{q j}$ is the vibrational frequency, and $e_{q j}$ is the eigenvector for phonon branch $j$. We also compute the contribution of each atomic species. In our calculations the cell volume varies by $1 \%$ with respect to the relaxed volume. Finally, magnetic ordering has been studied with spin-polarized formalism by setting a starting magnetization to break the symmetry and to provide a starting point for self-consistency. Both ferromagnetic and antiferromagnetic orders have been explored.

\section{Crystal Structure}

Mawsonite $\mathrm{Cu}_{6} \mathrm{Fe}_{2} \mathrm{SnS}_{8}$ is a typical copper-iron sulfide with a complex unit cell composed of 17 atoms (space group $P \overline{4} m 2, \mathrm{~N}^{\circ} 115$ ). Similarly to colusite, chalcopyrite and kesterite, mawsonite crystallizes in a tetragonal structure derived from the zinc-blende: corner sharing $\mathrm{SnS}_{4}$ and $\mathrm{CuS}_{4}$ distorted tetrahedrons, centered around the cations $\mathrm{Sn}(1 a)$ and $\mathrm{Cu}(2 g$ and $4 i$ ), are stacking along the same axis, whereas regular $\mathrm{FeS}_{4}(1 b$ and $1 c$ ) tetrahedrons share edges. In Figure 1 the green $\mathrm{Cu}(4 \mathrm{i})$ atoms highlight a layered sublattice that builds a continous network. We report in Table 1 the optimized lattice and structural parameters for the psedopotentials used (and experimental data for comparison). The theoretical volumes 
obtained with a variable cell calculation are within $6 \%$ of the experimental values.

Table 1: Structural parameters determined theoretically for the mawsonite structure. Experimental data from Szymanski ${ }^{44}$ are in excellent agreement with the calculations. Lattice parameters were determined using PBESol pseudopotentials, $\mathrm{a}=7.476 \AA$ and $\mathrm{c}=5.178 \AA$, and PBE pseudopotentials $\mathrm{a}=7.556 \AA$ and $\mathrm{c}=5.356 \AA$. The experimental values given by the American Mineralogist Crystal Structure Database ${ }^{45}$ are $a=7.603 \AA$ and $c=5.358 \AA$.

\begin{tabular}{cccc}
\hline & $\mathrm{x}$ & $\mathrm{y}$ & $\mathrm{z}$ \\
\hline \hline $\mathrm{Sn}(1 \mathrm{a})$ & 0.0000 & 0.0000 & 0.0000 \\
$\mathrm{Cu}(2 \mathrm{~g})$ & 0.5000 & 0.0000 & 0.0001 \\
$\mathrm{Cu}(4 \mathrm{i})$ & 0.2471 & 0.2471 & 0.5000 \\
$\mathrm{Fe}(1 \mathrm{~b})$ & 0.5000 & 0.5000 & 0.0000 \\
$\mathrm{Fe}(1 \mathrm{c})$ & 0.5000 & 0.5000 & 0.5000 \\
$\mathrm{~S}(4 \mathrm{j})$ & 0.2625 & 0.0000 & 0.2533 \\
$\mathrm{~S}(4 \mathrm{k})$ & 0.2565 & 0.5000 & 0.2487
\end{tabular}

Although Zhang et al. have reported the synthesis and thermoelectric properties of mawsonite ${ }^{27}$ careful examination of the powder X-ray diffraction data presented by these authors highlights major discrepancies between their experimental and calculated diffraction patterns, see Figure 2. According to Szymanski, ${ }^{44}$ mawsonite crystallises in a superstructure of sphalerite, and previously reported powder diffraction patterns of mawsonite show characteristic superstructure peaks at $2 \theta$ values of $16.5^{\circ}(110), 20.3^{\circ}(101), 23.4^{\circ}(200)$ and $31.1^{\circ}$ (211) ( $\mathrm{Cu} \mathrm{K} \alpha$ radiation $){ }^{46}$ which are in excellent agreement with the calculated pattern using the reported crystal structure. ${ }^{44}$ These characteristic superstructure peaks are absent in the experimental powder diffraction pattern reported by Zhang et al., ${ }^{27}$ which instead contains markedly different superstructure peaks, presumably corresponding to another superstructure of sphalerite. More recently, these authors have identified the material prepared by them as a mixture of phases, rather than pure mawsonite. ${ }^{47}$ 


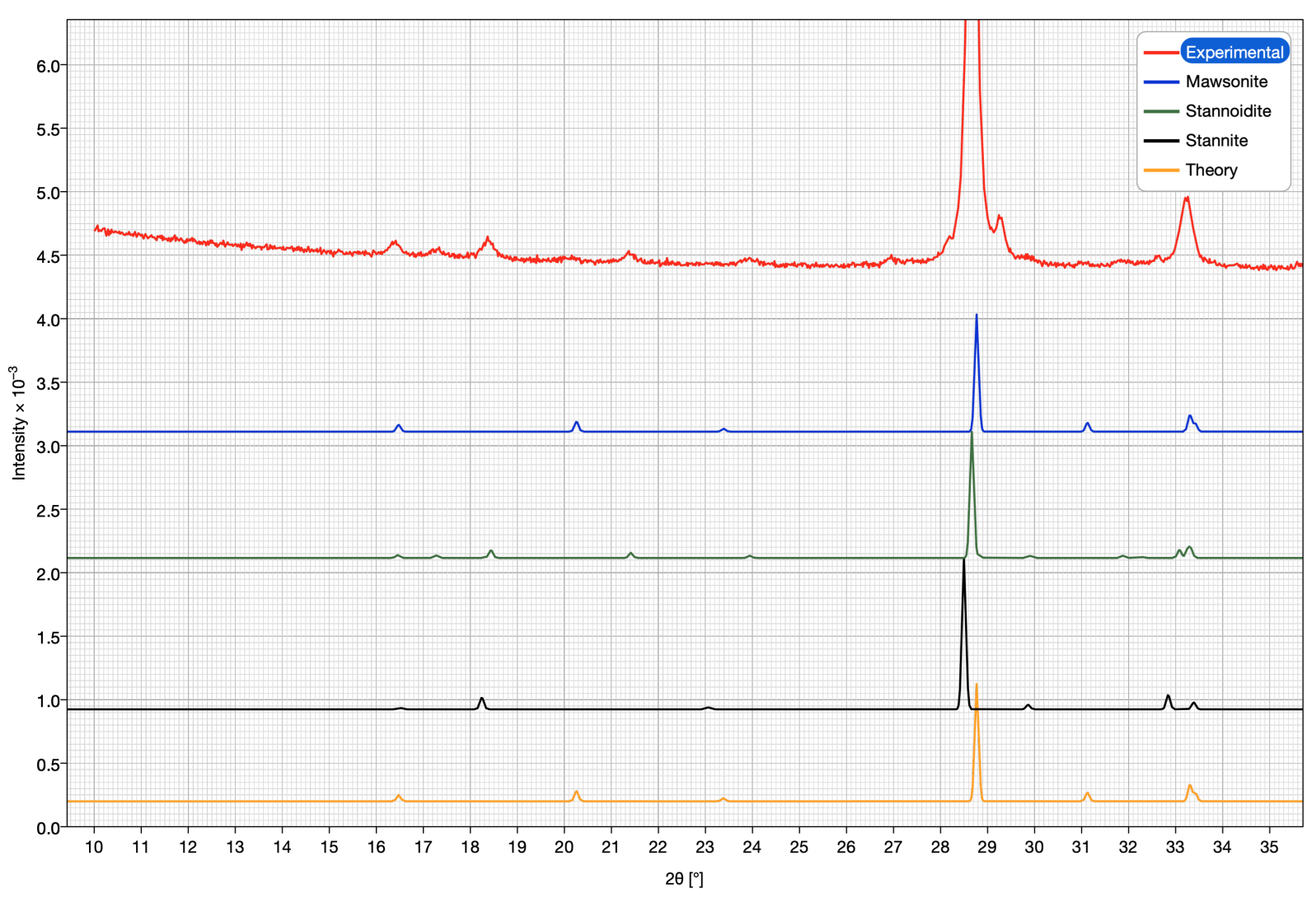

Figure 2: X-ray powder diffraction patterns for the DFT relaxed (yellow) and experimental (blue) mawsonite $\mathrm{Cu}_{6} \mathrm{Fe}_{2} \mathrm{SnS}_{8}$ as reported by Szymanski et al. ${ }^{44}$ We also report the pattern of a sample (red) with composition $\mathrm{Cu}_{6} \mathrm{Fe}_{2} \mathrm{SnS}_{8}$, prepared under similar conditions to those reported by Zhang et al. ${ }^{27}$ For reference we include the experimental patterns for stannite (black) and stannoidite (green); these structures are from the American Mineralogist Crystal Structure Database. ${ }^{45}$ 

i.e. $[\mathrm{Cu}] /[\mathrm{M}]>1$, exhibit p-type conductivity. ${ }^{20,48,49}$ Indeed, the ability of the univalent copper to accomodate tetrahedral coordination and to form a tridimensional framework is favorable to the delocalization of p-type carriers. These considerations, together with charge balance, suggest: $\mathrm{Cu}^{+1}, \mathrm{Fe}^{+3}, \mathrm{Sn}^{+4}, \mathrm{~S}^{-2}$; these are confirmed by Mössbauer experiments where mawsonite is shown to contain only ferric iron. ${ }^{50}$ To address the general features of the electronic structure of $\mathrm{Cu}_{6} \mathrm{Fe}_{2} \mathrm{SnS}_{8}$, we computed the spin polarized band structure and the projected DOS in Figure 3, where spin polarization is considered to properly describe the transition metal $\mathrm{Fe}^{3+}$. Mawsonite is a semiconductor which experimentally tends to exhibit p-type transport, therefore our interest is in the upper valence band (VB) mainly composed of strongly hybridized $\mathrm{S} p$-orbitals and $\mathrm{Cu} d$-orbitals. More specifically, the projected density of states resolved by site shows a major contribution from $\mathrm{Cu}(4 \mathrm{i})$ with respect to $\mathrm{Cu}(2 \mathrm{~g})$ in the energy range close to Fermi, see Figure S1a Supporting Information. This feature suggests that $\mathrm{Cu}(4 \mathrm{i})-\mathrm{S}$ bonding builds a $2 \mathrm{D}$ conductive network with negligible contribution from other species. Conductive networks have been observed in other diamond-like coppersulfides $^{51}$ where $\mathrm{Cu}-(\mathrm{S}, \mathrm{Se})$ atoms determine the relevant electronic states for the transport properties. Despite the strong localized character of the $\mathrm{Cu} d$-orbitals, the hybridization with S $p$-orbitals favors the delocalization of p-type carrier and provides heavy but still conductive bands with remarkable features for thermoelectric transport; near the Fermi level, Figure 3 points to a multi-valley character with large effective masses especially at M, Z, R high symmetry points of the Brillouin zone, two features that contribute to a large Seebeck coefficient and enhanced electrical conductivity. In this perspective, out-of-network atoms could serve as doping/alloying sites to tune other properties without affecting the conducting states. Also, Figure 3 displays hybridized iron $d$ - and sulfur $p$-orbitals at the bottom of conduction band $(\mathrm{CB})$, where a single and relatively heavy band singles out, possibly leading to spin polarized n-type transport. 
The band structure in Figure 3 corresponds to an antiferromagnetic ordered ground
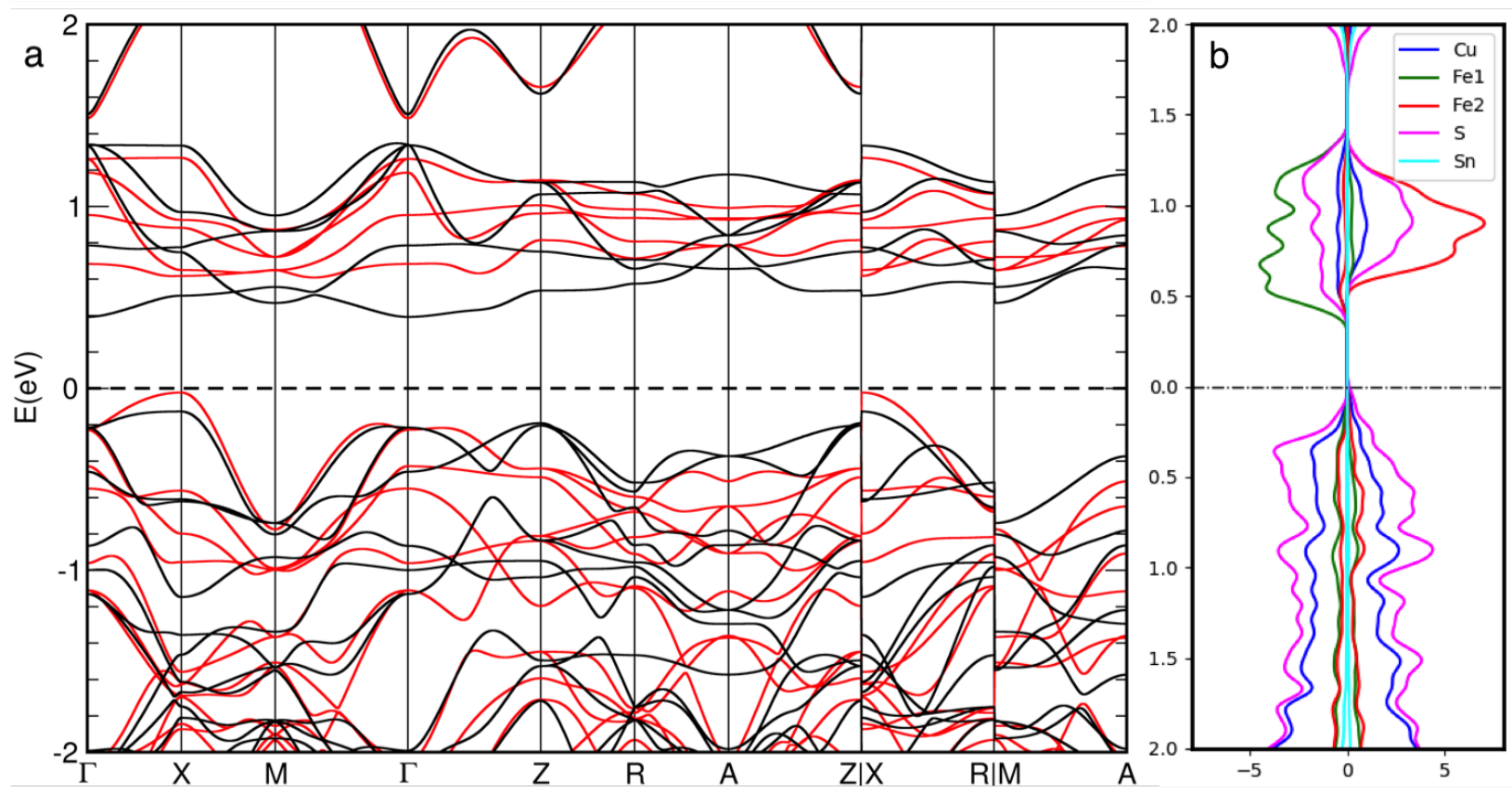

Figure 3: Spin polarized electronic band structure (a) and atom projected density of states (b) of $\mathrm{Cu}_{6} \mathrm{Fe}_{2} \mathrm{SnS}_{8}$. Spin up (down) channel is represented in red (black). Hubbard $U$ corrections are computed self-consistently within the ACBN0 approach: $\mathrm{U}(\mathrm{Cu})=6.595 \mathrm{eV}$, $\mathrm{U}(\mathrm{Fe}$ in $1 b)=0.551 \mathrm{eV}, \mathrm{U}(\mathrm{Fe}$ in $1 c)=0.518 \mathrm{eV}, \mathrm{U}(\mathrm{S})=0.952 \mathrm{eV}, \mathrm{U}(\mathrm{Sn})=0.002 \mathrm{eV}$

state. This configuration is energetically favored with respect to the ferromagnetic one, $E_{F M}-E_{A M}=0.16 \mathrm{eV}$ per formula unit, and the large energy difference suggests a high ordering temperature, in agreement with experimental results for another tetragonal sulfide, the chalcopyrite $\mathrm{CuFeS}_{2}\left(\mathrm{~T}_{\text {Neel }}=823 \mathrm{~K}\right){ }^{52,53}$. Further, spin majority and minority bands have direct and indirect gap respectively, $\Delta E^{\uparrow}=0.6 \mathrm{eV}$ and $\Delta E^{\downarrow}=0.4 \mathrm{eV}$. The two spin channels, up (red) and down (black), are shifted one with respect to the other, thus producing a spin polarized isolated band in the bottom of CB. The shift arises because of the asymmetry in the magnetic moment distribution between the two iron sites, $\mu_{F e 1}=3.01 \mu_{B}$ and $\mu_{F e 2}=-2.89 \mu_{B}$. This effect is highlighted also by different profiles in the projected DOS (compare green to purple curve in Figure 3b), the values of the Hubbard correction, $U$, see Figure 3) as well as in local spin polarization isosurface in Figure 1. The unusually low value of the magnetic moments for $\mathrm{Fe}^{3+}$ are due to deviations from the ionic picture ${ }^{54}$ 
which are observed by inspecting the electron localization factor, Figure 4.

The features revealed in the band structure analysis have a correspondence in the dependence on temperature of the Seebeck coefficient $S$ and electrical conductivity $\sigma$, displayed in Figure 5. For both the VB and CB, $\sigma$ increases with temperature as it is usual for semiconductors. Seebeck coefficients, which do not depend on the choice of the constant relaxation time, are predicted in excess of $250 \frac{\mu V}{K}$ for light p-type doping at $500 \mathrm{~K}$. Comparable negative values for light n-type were obtained. As mentioned in the computational method, our Boltzmann transport calculations consider a constant scattering time $\tau$ across the range of temperature. The conductivities (with our assumption of $\tau=10^{-15} \mathrm{~s}$ ) are monotonically increasing with temperature and quite small. The smaller values observed for the $\mathrm{CB}$ are due to the presence of the "isolated" single band at the botton of the conduction band. Due to the uncertainity on the value of the relaxation time, conductivity predictions should be taken with caution. Indeed $\tau=10^{-14} \mathrm{~s}$ will make mawsonite much more promising for thermoelectric applications. We noticed, however, that with $\tau=10^{-15} \mathrm{~s}$, despite the difference in the crystal structure pointed out when discussing the crystal structure, the computed $S$ and $\sigma$ are in agreement with experimental data reported in Zhang et al. ${ }^{27}$ once the chemical potential is shifted toward the valence band, $\mu=-0.11 \mathrm{eV}$. This may indicate that the transport properties are mainly determined by common features such as a similar conductive network (Cu-S atoms).

\section{Vibrational properties and lattice thermal conductivity}

The phonon dispersion and atom-resolved phonon DOS computed by first principles are in Figure 6. Even though calculations within the harmonic approximation do not provide any direct information on the thermal conductivity, they constitute a starting point to discuss heat transport, especially the acoustic modes with larger velocity. Low frequencies optic 
Figure 4: Contour of the electron localization factor shown on selected planes. Contour plot of the electron localization factor (ELF) shown on selected planes. Atoms are colored as in Figure 1. Values for the ELF range between 0 and 1: $E L F=0.5$ (corresponding to the blue contours) indicates free electron behavior and $E L F=1.0$ (red contour) indicates perfect localization. Values smaller than 0.5 are less significant and usually point to small local electron density. Substantial localization is observed in proximity of S and one Fe (1b). Directional covalent bonds are observed between the $\mathrm{Sn}$ and the $\mathrm{S}$. The plane containing $\mathrm{Cu}(4 \mathrm{i})$ exhibit pockets of "free electron" $(\mathrm{ELF}=0.5)$ potentially associated with larger metallicity. These considerations agree with the COHP analysis shown in the Figure S2 Supporting Information. 


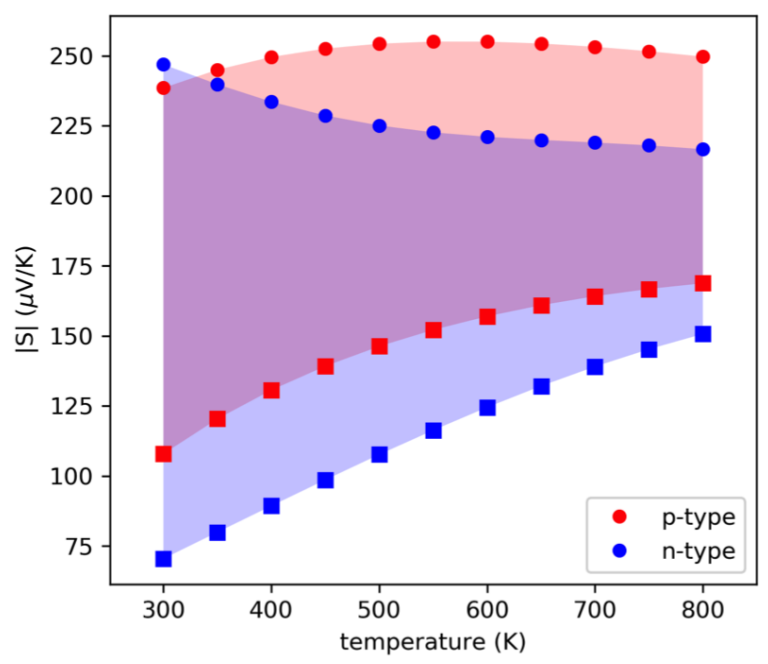

Figure 5: Temperature dependence of electrical conductivity (left panel) and absolute value of the Seebeck coefficient (right panel). The red and blue shade highlight the range of values taken by these quantities as the chemical potential moves inside valence and conduction band, respectively. Red (blue) dots represent the top (bottom) of the valence (conduction) band, while red (blue) squares refers to a chemical potential in the valence (conduction) band $(\Delta \mu=0.1 \mathrm{eV})$. Resistivities were computed assuming $\tau=10^{-15} \mathrm{~s}$.

modes sharing the frequency window with acoustic vibrations are a source of phonon scattering and affects the thermal transport. Despite the traditional use of heavy ions as rattlers to open scattering channels, ${ }^{17,55}$ recently similar performance have been reported in materials containing light elements, such as colusite $\mathrm{Cu}_{26} \mathrm{~V}_{2} \mathrm{Sn}_{6} \mathrm{~S}_{32}{ }^{23}$ and $\mathrm{BiOCuQ}(\mathrm{Q}=\mathrm{Se}, \mathrm{Te}) .{ }^{15}$ Low frequency modes centered around 40-60 $\mathrm{cm}^{-1}$ appear in mawsonite phonon dispersion in Figure 6. The atom resolved DOS suggests that the $\mathrm{Cu}$ contribution at low frequency exceeds the ones from other species. Due to the light $\mathrm{Cu}$ mass, we must assume very weak bonds near $\mathrm{Cu}$ sites. In order to evaluate the role of $\mathrm{Cu}$ in lowering the lattice thermal conductivity, we use the quasi-harmonic apporximation to estimate the degree of anharmonicity of mawsonite through the mode-resolved Grüneisen parameter $\gamma_{q j}$, see Eq. [6], and its atoms projection as a function of the frequency. Our results, displayed in Figure 7, clearly indicate that modes are highly anharmonic throughout the spectrum, but specifically in the low frequency range $50-175 \mathrm{~cm}^{-1}$, see Figure $7 \mathrm{a}$, where the major contribution comes from $\mathrm{Cu}$ 


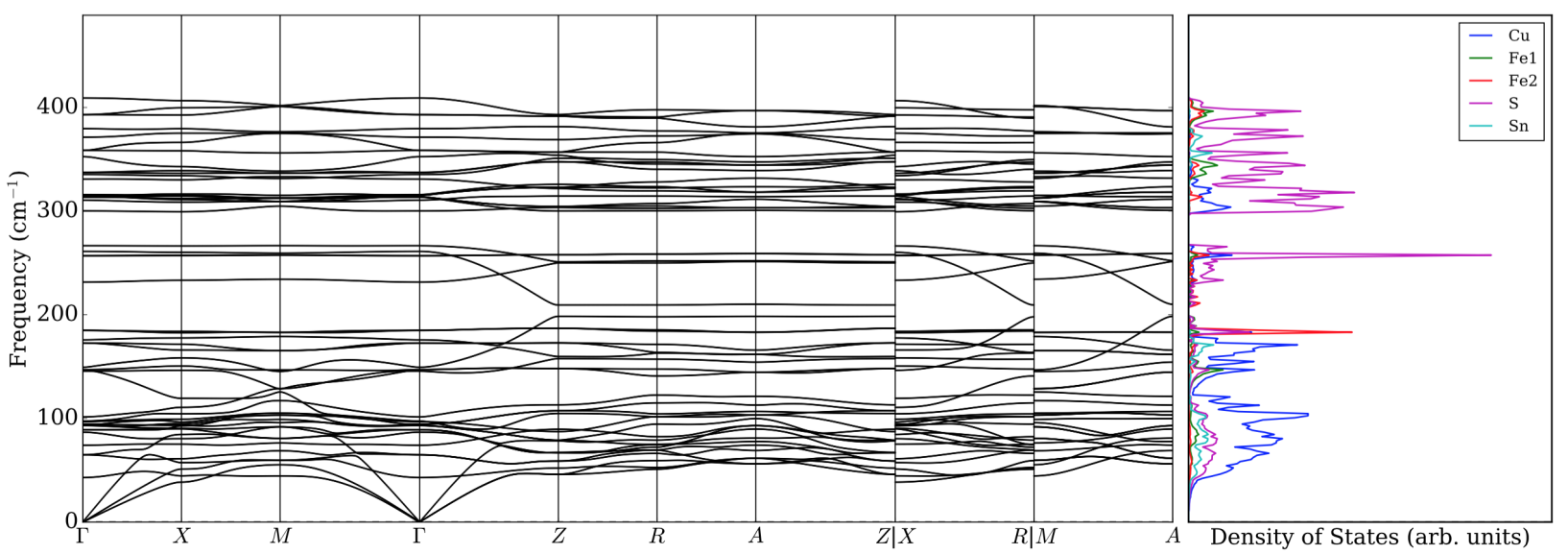

Figure 6: Phonon dispersion and atom-projected vibrational density of states of $\mathrm{Cu}_{6} \mathrm{Fe}_{2} \mathrm{SnS}_{8}$. Notice the prominent role of $\mathrm{Cu}$ at low frequency; optic modes at frequency lower than 100 cm-1 contribute to scattering phenomena that lower the lattice thermal conductivity.

ions, see Figure 7b. This finding also support the conjecture that $\mathrm{Cu}$ has a special role as a weakly bonded component in mawsonite.

The temperature dependence of the total thermal conductivity $\kappa$ (squares) and its electronic $\kappa_{e l}$ (dots) and lattice (green dots) components $\kappa_{\text {latt }}$ is presented in Figure 8. $\kappa_{t o t}$ decreases on the whole temperature range from 0.6 to $0.23 \mathrm{~W} \mathrm{~m}^{-1} \mathrm{~K}^{-1}$, and is dominated by the lattice component. Similar behavior is observed for $\mathrm{p}$ - and n-type doping. Based on the analysis of the vibrational spectra, the origin of this low thermal conductivity is mainly attributed to the scattering effects induced by the disordered $\mathrm{Cu}$, that scatter long wavelength phonons. This is confirmed by the sound velocities for acoustic phonons reported in Table S2 Supporting Information. Mawsonite's values appear sensibly higher than the ones reported for SnSe. ${ }^{56}$ The close values for the thermal conductivities for the two compounds suggest that anharmonic effects in mawsonite plays a major role in getting a thermal conductivity as low as for SnSe. In this picture low thermal conductivity depends on the coexistence of weakly bonded quasi-liquid subsystems within a crystalline matrix. This is different to the traditional phonon-glass electron-crystal paradigm as understood in cage-materials, where instead there is a disordered set of non-interacting point defects. Mawsonite has weakly-bonded component $(\mathrm{Cu})$ whose mobility is suppressed due to the absence 

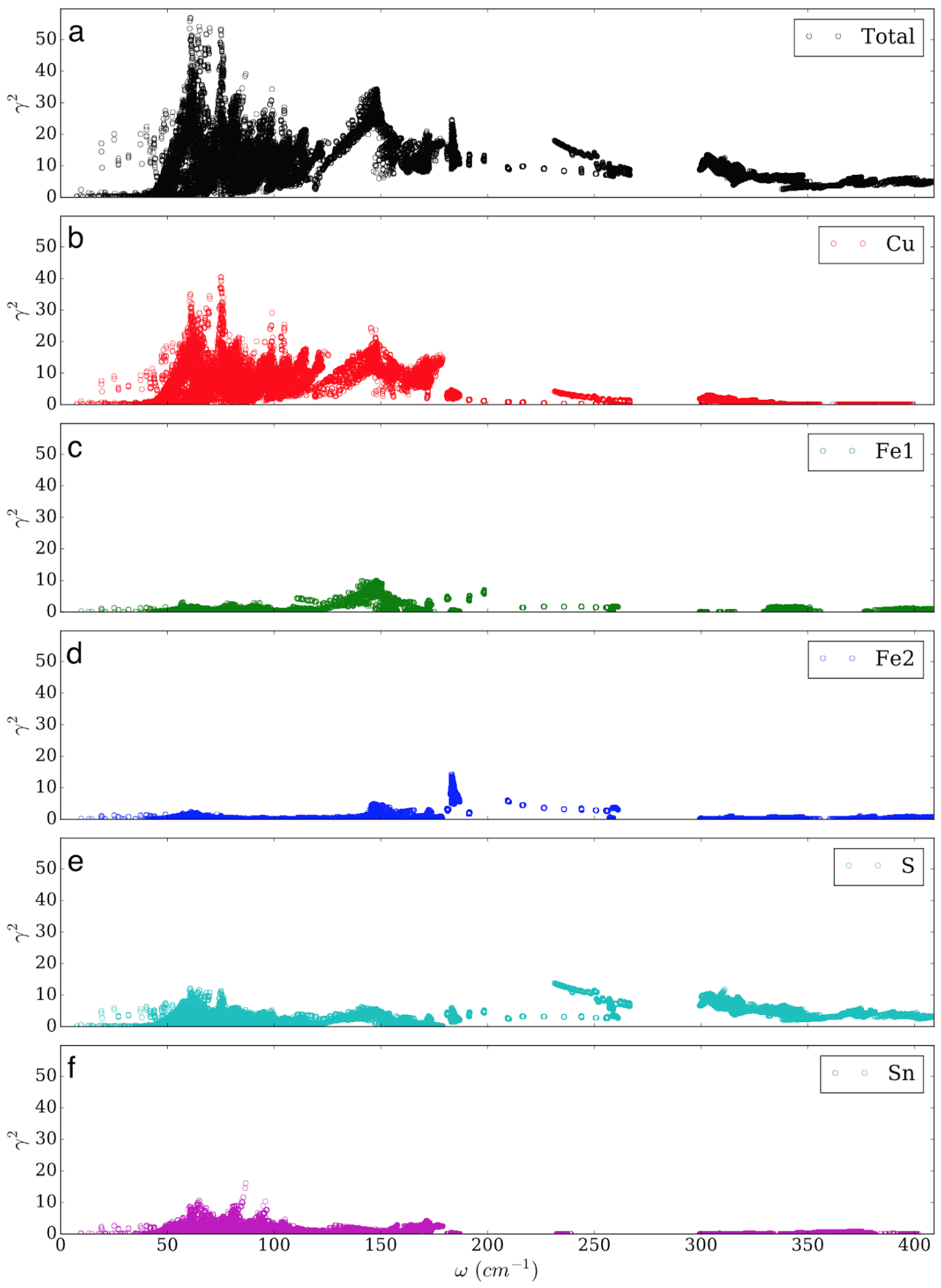

Figure 7: (a) Mode resolved Grüneisen parameter $\gamma_{q j}$ as a function of mode frequency; (b-f) contribution of each atomic species to the total Grüneisen as a function of the mode frequency. 
of a suitable diffusion path. This is definitely relevant for thermoelectric application, given the detrimental effect of ion migration to the device.

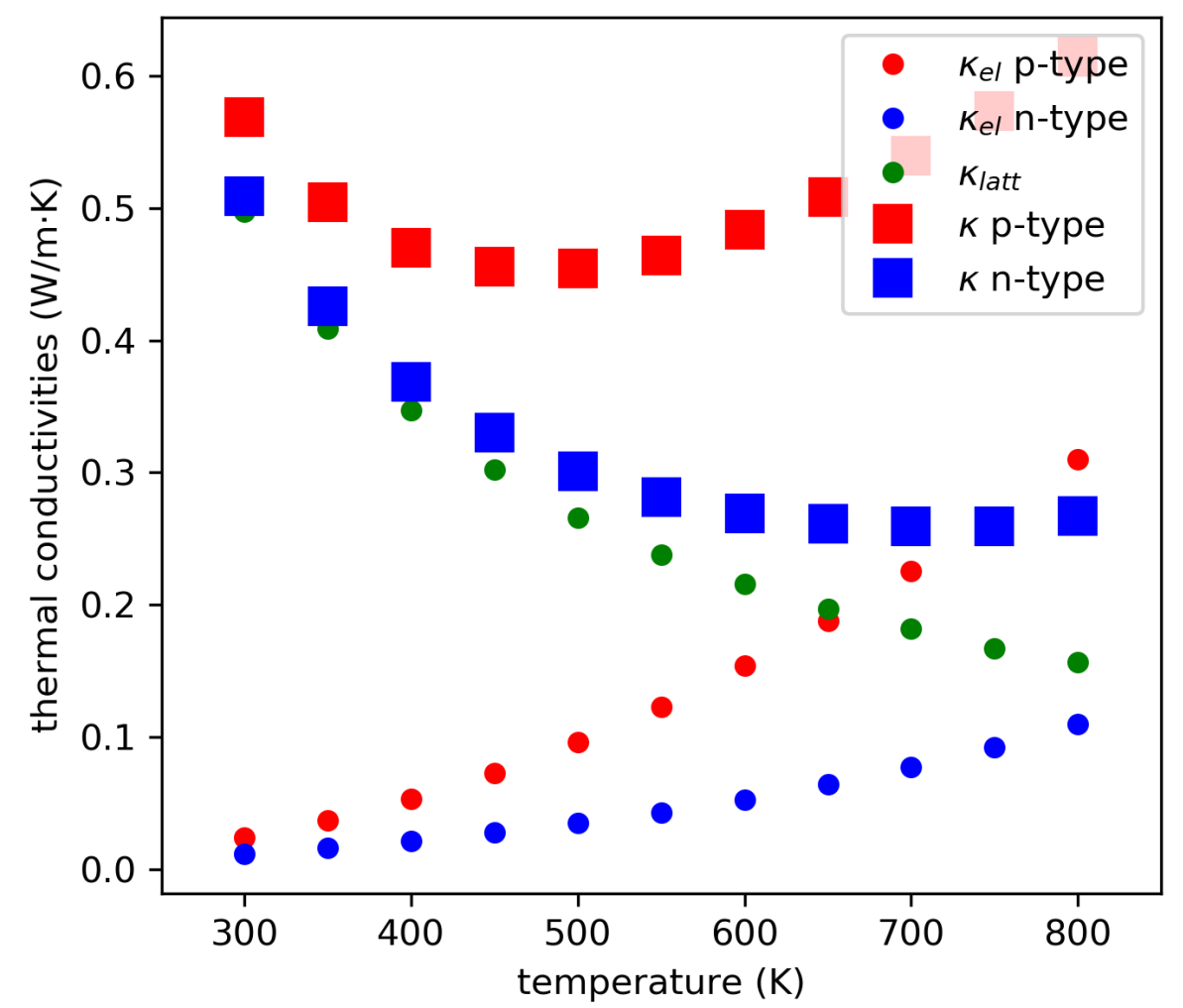

Figure 8: Individual components and total thermal conductivity computed according to the Debye-Callaway model as a function of temperature for different chemical potentials: electronic component $\kappa_{e l}$ calculated at top of the valence band (red dots) and at bottom of the conduction band (blue dots), lattice component $\kappa_{\text {lat }}$ (green dots) and total thermal conductivity $\kappa_{e l}+\kappa_{\text {lat }}$ for p-type mawsonite (red squares) and n-type (blue squares).

\section{Chemical substitutions}

Chemical replacement in prototypical materials has been extensively used to optimize the functional properties of thermoelectric materials. Starting from the mawsonite structure, we substituted Fe with $\mathrm{Ni}, \mathrm{S}$ with $\mathrm{Se}$, Te, and $\mathrm{Sn}$ with Ge, Pb. We have checked the thermodynamical stability of the resulting compounds by computing the formation energy 
$E_{f}$ as the difference between the total energy of the substituted compound and the total energy of each component in the crystalline form: $E_{f}=E\left[C u_{6} X_{2} Y Z_{8}\right]-6 E[C u]-2 E[X]-$ $E[Y]-8 E[Z]$. All the compounds are shown to be stable, as reported in the Table S1 Supporting Information. The band structure and projected DOS of the pristine mawsonite are displayed in Figure S3 Supporting Information on a larger energy window, where it is more evident that the conductive network formed by $C u S$ tetrahedra defines the manifold at the bottom of the valence band and the one in the conduction band above $3 \mathrm{eV}$. In between these energy windows, we observe the contribution of other cations: a manifold of flat bands from the $d$-orbitals of the transition metal ions (Fe) strongly hybridized with $\mathrm{S} p$-orbitals, and a highly dispersive band arising from hybridization of Sn $s$-orbital with $\mathrm{S} p$-orbitals. The Ni substitution, see Figure S4 Supporting Information, changes the magnetic character of the pristine mawsonite and shifts the d-manifold down toward the valence band forming a metallic band structure for all the Ni-compounds. Similarly the substitutions of Sn mainly affect the $s$-band that is shifted from the region close to $3 \mathrm{eV}$ to the transition metal manifold as one moves from Ge to $\mathrm{Pb}$. The isolated bands is obtained as long as there is energy difference between $s$ - and $p$ - orbitals of the IV group element, and such difference increases moving along the IV group elements, from Ge to $\mathrm{Pb}$. Finally, the effect of the chalcogenide substitution: from band structures (Figure S5-S20 Supporting Information), we observe the common trend that sulfides have generally a larger band gap (more ionicity), that decreases with Se, Te substitution due to the stronger hybridization between $p$ - and $d$-orbitals. Sn substitutions preserve the features we previously pointed out for mawsonite (such as flat band and valley degeneracy).

Overall the semiconductor character is preserved in $\mathrm{Cu}_{6} \mathrm{Fe}_{2} \mathrm{SnSe}_{8}, \mathrm{Cu}_{6} \mathrm{Fe}_{2} \mathrm{GeX}_{8}$ with $\mathrm{X}=\mathrm{S}$, Se, and Te, as well as in $\mathrm{Cu}_{6} \mathrm{Fe}_{2} \mathrm{PbS}_{8}$. All the other cases, we investigated, result in a metallic band structure due to a relative shift of $\mathrm{Cu}-\mathrm{S}$ manifold and transition metal bands. 


\section{Conclusions}

Materials with the mawsonite structure, namely $\mathrm{Cu}_{6} \mathrm{X}_{2} \mathrm{YZ}_{8}$ with $\mathrm{X}=\mathrm{Fe}, \mathrm{Ni}, \mathrm{Y}=\mathrm{Ge}, \mathrm{Pb}$, and $\mathrm{Z}=\mathrm{S}$, Se, Te, have been studied from the point of view of their thermoelectric potentials for low-cost and environmentally sound, low-grade energy recovery systems. We used state-of-the-art first principles methodologies based on self-consistent $\mathrm{DFT}+\mathrm{U}$ to compute band structure, projected density of states (DOS), and electronic transport coefficients. In addition, in selected cases, we determined the phonon dispersions and the lattice thermal conductivity. Our findings indicate that the low thermal conductivity is due to low-frequency vibrational modes of the copper atoms that activate scattering channels for acoustic phonons thus affecting the heat transport. As corroborated by the Grüneisen calculations, mawsonite has a quasi-liquid disordered component $(\mathrm{Cu})$ within the crystalline matrix but do not present ion migration due to the absence of suitable diffusion paths. The analysis of the transport coefficients supported by band structure calculation highlights a $2 \mathrm{D}$ conductive network built by $\mathrm{Cu}(4 \mathrm{i})-\mathrm{S}$ bonds which determines the TE properties of the p-type mawsonite. The covalency of the $\mathrm{Cu}(4 \mathrm{i})-\mathrm{S}$ bonding causes an asymmetry in the distribution of the Fe magnetic moments, that originates an isolated band possibly leading to a spin-polarized n-type transport. Finally, chemical substitution of out of network species shifts the manifolds in the conduction band and change the compound character from semiconducting to metallic, thus consequently affecting the magnetic order. Overall, as displayed in Figure 9 , the combination of good electronic transport and low thermal conductivity provides promising TE performance in the medium temperature range with a ZT value that point to 0.5 @ $750 \mathrm{~K}$.

\section{Acknowledgement}

We thank Andrew Supka for technical assistance and useful discussions. We thank Virginia Carnevali for graphic assistance. The members of the AFLOW Consortium (http://www.aflow.org) acknowledge DOD-ONR support DOD-ONR (Grants N000141310635 and N000141512266). 

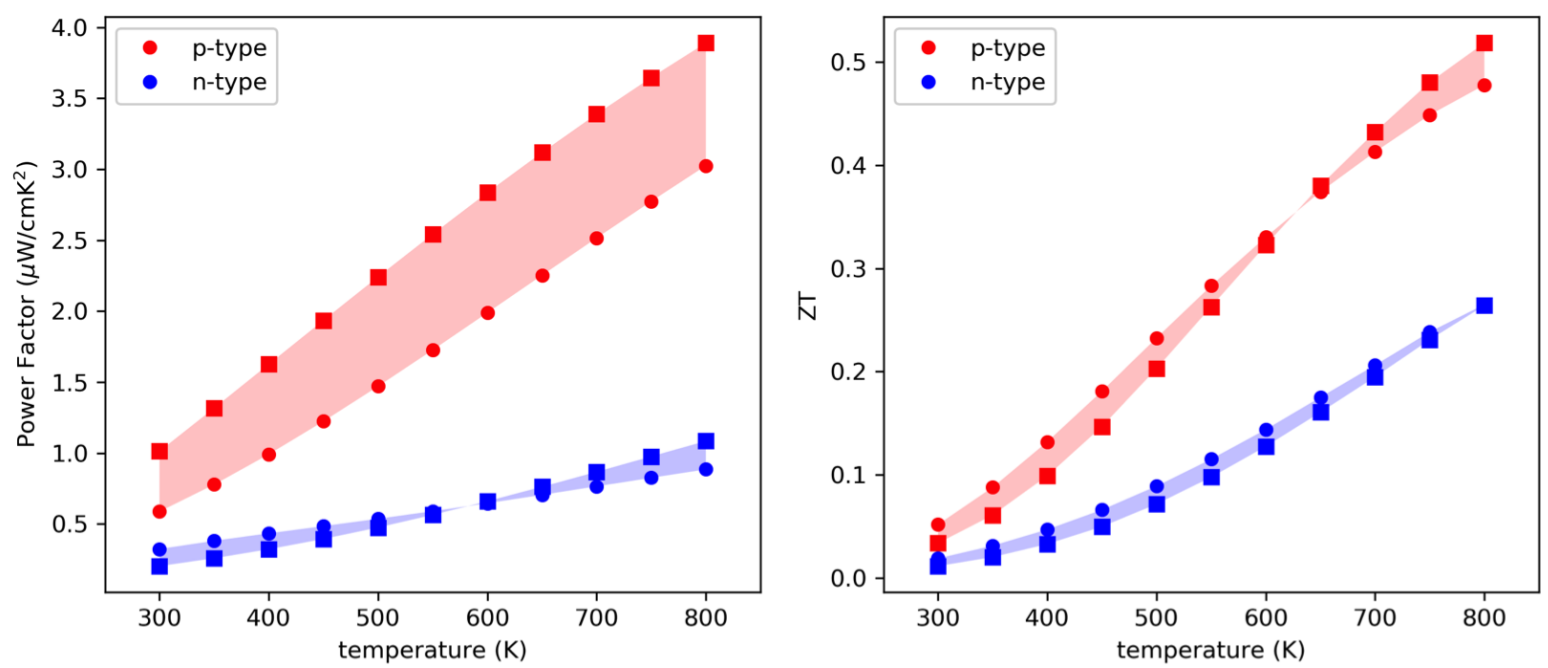

Figure 9: Temperature dependence of power factor (left pane) and ZT (right panel). The red and blue shade highlight the range of values taken by these quantities as the chemical potential moves inside valence and conduction band, respectively. Red (blue) dots represent the top (bottom) of the valence (conduction) band, while red (blue) squares refers to a chemical potential in the valence (conduction) band $(\Delta \mu=0.1 \mathrm{eV})$.

Finally, we acknowledge the High Performance Computing Center at the University of North Texas and the Texas Advanced Computing Center at the University of Texas, Austin.

\section{Supporting Information Available}

Projected DOS contribution of $\mathrm{Cu}(4 \mathrm{i})$ and $\mathrm{Cu}(2 \mathrm{~g})$ species, spin majority and minority contribution to the total DOS, formation energies for all the considered compounds, crystal orbital Hamilton population, sound velocities for mawsonite, parameters for the Debye-Callaway model computed from first-principles, bandstructures and projected DOS of the pristine mawsonite and of all the considered compounds with the mawsonite structure, $\mathrm{Cu}_{6} \mathrm{X}_{2} \mathrm{YZ}_{8}$ with $\mathrm{X}=\mathrm{Fe}, \mathrm{Ni}, \mathrm{Y}=\mathrm{Ge}, \mathrm{Pb}$, and $\mathrm{Z}=\mathrm{S}$, Se, Te.

This material is available free of charge via the Internet at http://pubs.acs.org/. 


\section{References}

(1) Kajikawa, T. Thermoelectric power generation system recovering industrial waste heat. Thermoelectrics Handbook: Macro to Nano, CRC/Taylor \& Francis., DM Rowe 2006, 50-1.

(2) https://www . energy .gov, DepartmentofEnergy, USA.

(3) Zevenhoven, R.; Beyene, A. The relative contribution of waste heat from power plants to global warming. Energy 2011, 36, 3754-3762.

(4) Caillat, T.; Fleurial, J.-P.; Borshchevsky, A. Preparation and thermoelectric properties of semiconducting $\mathrm{Zn}_{4} \mathrm{Sb}_{3}$. J. Phys. Chem. Solids 1997, 58, 1119 - 1125.

(5) Hsu, K. F.; Loo, S.; Guo, F.; Chen, W.; Dyck, J. S.; Uher, C.; Hogan, T.; Polychroniadis, E. K.; Kanatzidis, M. G. Cubic $\mathrm{AgPb}_{\mathrm{m}} \mathrm{SbTe}_{2+\mathrm{m}}$ : Bulk thermoelectric materials with high figure of merit. Science 2004, 303, 818-821.

(6) Liu, W.; Tan, X.; Yin, K.; Liu, H.; Tang, X.; Shi, J.; Zhang, Q.; Uher, C. Convergence of conduction bands as a means of enhancing thermoelectric performance of $n$-type $\mathrm{Mg}_{2} \mathrm{Si}_{1-x} \mathrm{Sn}_{x}$ solid solutions. Phys. Rev. Lett. 2012, 108, 166601.

(7) Poudeu, P.; D’Angelo, J.; Downey, A.; Short, J.; Hogan, T.; Kanatzidis, M. High thermoelectric figure of merit and nanostructuring in bulk p-type $\mathrm{Na}_{1-x} \mathrm{~Pb}_{m} \mathrm{Sb}_{y} \mathrm{Te}_{m+2}$. Angew. Chem. Int. Ed. 2006, 45, 3835-3839.

(8) Tan, G.; Shi, F.; Hao, S.; Zhao, L.-D.; Chi, H.; Zhang, X.; Uher, C.; Wolverton, C.; Dravid, V.; Kanatzidis, M. Non-equilibrium processing leads to record high thermoelectric figure of merit in PbTe-SrTe. Nat. Commun. 2016, \%, 12167.

(9) Pei, Y.; Lensch-Falk, J.; Toberer, E. S.; Medlin, D. L.; Snyder, G. J. High thermoelectric performance in PbTe due to large nanoscale $\mathrm{Ag}_{2}$ Te precipitates and La doping. Adv. Funct. Mater. 2011, 21, 241-249. 
(12) Yang, J.; Xi, L.; Qiu, W.; Wu, L.; Shi, X.; Chen, L.; Yang, J.; Zhang, W.; Uher, C.; Singh, D. J. On the tuning of electrical and thermal transport in thermoelectrics: an integrated theory-experiment perspective. Npj Comput. Mater. 2016, 2, 15015.

(13) Pavan Kumar, V.; Supka, A. R.; Lemoine, P.; Lebedev, O. I.; Raveau, B.; Suekuni, K.; Nassif, V.; Al Rahal Al Orabi, R.; Fornari, M.; Guilmeau, E. High power factors of thermoelectric colusites $\mathrm{Cu}_{26} \mathrm{~T}_{2} \mathrm{Ge}_{6} \mathrm{~S}_{32}(\mathrm{~T}=\mathrm{Cr}, \mathrm{Mo}, \mathrm{W})$ : toward functionalization of the conductive "Cu-S" Network. Adv. Energy Mater. 2019, 9, 1803249.

(14) Lai, W.; Wang, Y.; Morelli, D. T.; Lu, X. From bonding asymmetry to anharmonic rattling in $\mathrm{Cu}_{12} \mathrm{Sb}_{4} \mathrm{~S}_{13}$ tetrahedrites: when lone-pair electrons are not so lonely. Adv. Funct. Mater. 2015, 25, 3648-3657.

(15) Vaqueiro, P.; Al Orabi, R. A. R.; Luu, S. D.; Guelou, G.; Powell, A. V.; Smith, R.; Song, J.-P.; Wee, D.; Fornari, M. The role of copper in the thermal conductivity of thermoelectric oxychalcogenides: do lone pairs matter? Phys. Chem. Chem. Phys. 2015, 17, 31735-31740.

(16) Venkatasubramanian, R.; Silvola, E.; Colpitts, T.; O'Quinn, B. Materials for Sustainable Energy; Co-Published with Macmillan Publishers Ltd, UK, 2012; pp 120-125.

(17) Shi, X.; Chen, L.; Uher, C. Recent advances in high-performance bulk thermoelectric materials. Int. Mater. Rev. 2016, 61, 379-415.

(18) Poudel, B.; Hao, Q.; Ma, Y.; Lan, Y.; Minnich, A.; Yu, B.; Yan, X.; Wang, D.; Muto, A.; Vashaee, D.; Chen, X.; Liu, J.; Dresselhaus, M. S.; Chen, G.; Ren, Z. 
High-Thermoelectric Performance of Nanostructured Bismuth Antimony Telluride Bulk Alloys. Science 2008, 320, 634-638.

(19) He, Y.; Day, T.; Zhang, T.; Liu, H.; Shi, X.; Chen, L.; Snyder, G. J. High thermoelectric performance in non-toxic earth-abundant copper sulfide. Adv. Mater. 2014, 26, 39743978.

(20) Xu, L.; T., M. D.; Yi, X.; Fei, Z.; Vidvuds, O.; Hang, C.; Xiaoyuan, Z.; Ctirad, U. High performance thermoelectricity in earth-abundant compounds based on natural mineral tetrahedrites. Adv. Energy Mater. 2013, 3, 342-348.

(21) Suekuni, K.; Tsuruta, K.; Kunii, M.; Nishiate, H.; Nishibori, E.; Maki, S.; Ohta, M.; Yamamoto, A.; Koyano, M. High-performance thermoelectric mineral $\mathrm{Cu}_{12-\mathrm{x}} \mathrm{Ni}_{\mathrm{x}} \mathrm{Sb}_{4} \mathrm{~S}_{13}$ tetrahedrite. J. Appl. Phys. 2013, 113, 043712.

(22) Suekuni, K.; Kim, F. S.; Nishiate, H.; Ohta, M.; Tanaka, H. I.; Takabatake, T. Highperformance thermoelectric minerals: colusites $\mathrm{Cu}_{26} \mathrm{~V}_{2} \mathrm{M}_{6} \mathrm{~S}_{32}(\mathrm{M}=\mathrm{Ge}, \mathrm{Sn})$. Appl. Phys. Lett. 2014, 105, 132107.

(23) Bourgès, C.; Bouyrie, Y.; Supka, A. R.; Al Rahal Al Orabi, R.; Lemoine, P.; Lebedev, O. I.; Ohta, M.; Suekuni, K.; Nassif, V.; Hardy, V.; Daou, R.; Miyazaki, Y.; Fornari, M.; Guilmeau, E. High-performance thermoelectric bulk colusite by process controlled structural disordering. JACS 2018, 140, 2186-2195.

(24) Qiu, P.; Zhang, T.; Qiu, Y.; Shi, X.; Chen, L. Sulfide bornite thermoelectric material: a natural mineral with ultralow thermal conductivity. Energy Environ. Sci. 2014, 7, 4000-4006.

(25) Pavan Kumar, V.; Barbier, T.; Caignaert, V.; Raveau, B.; Daou, R.; Malaman, B.; Caër, G. L.; Lemoine, P.; Guilmeau, E. Copper hyper-stoichiometry: the key for the optimization of thermoelectric properties in stannoidite $\mathrm{Cu}_{8+x} \mathrm{Fe}_{3-x} \mathrm{Sn}_{2} \mathrm{~S}_{12}$. J. Phys. Chem. C 2017, 121, 16454-16461. 
(27) Zhang, R.-z.; Chen, K.; Du, B.; Reece, M. J. Screening for Cu-S based thermoelectric materials using crystal structure features. J. Mater. Chem. A 2017, 5, 5013-5019.

(28) Giannozzi, P.; Baroni, S.; Bonini, N.; Calandra, M.; Car, R.; Cavazzoni, C.; Ceresoli, D.; Chiarotti, G. L.; Cococcioni, M.; Dabo, I.; Corso, A. D.; de Gironcoli, S.; Fabris, S.; Fratesi, G.; Gebauer, R.; Gerstmann, U.; Gougoussis, C.; Kokalj, A.; Lazzeri, M.; Martin-Samos, L.; Marzari, N.; Mauri, F.; Mazzarello, R.; Paolini, S.; Pasquarello, A.; Paulatto, L.; Sbraccia, C.; Scandolo, S.; Sclauzero, G.; Seitsonen, A. P.; Smogunov, A.; Umari, P.; Wentzcovitch, R. M. QUANTUM ESPRESSO: a modular and open-source software project for quantum simulations of materials. J. Phys. Condens. Matter. 2009, 21, 395502 .

(29) Giannozzi, P.; Andreussi, O.; Brumme, T.; Bunau, O.; Nardelli, M. B.; Calandra, M.; Car, R.; Cavazzoni, C.; Ceresoli, D.; Cococcioni, M.; Colonna, N.; Carnimeo, I.; Corso, A. D.; de Gironcoli, S.; Delugas, P.; Jr, R. A. D.; Ferretti, A.; Floris, A.; Fratesi, G.; Fugallo, G.; Gebauer, R.; Gerstmann, U.; Giustino, F.; Gorni, T.; Jia, J.; Kawamura, M.; Ko, H.-Y.; Kokalj, A.; KÃCẼ̃ß̃̃ (Ekbenli, E.; Lazzeri, M.; Marsili, M.; Marzari, N.; Mauri, F.; Nguyen, N. L.; Nguyen, H.-V.; de-la Roza, A. O.; Paulatto, L.; Ponc⿱̃⿻上丨@, S.; Rocca, D.; Sabatini, R.; Santra, B.; Schlipf, M.; Seitsonen, A. P.; Smogunov, A.; Timrov, I.; Thonhauser, T.; Umari, P.; Vast, N.; Wu, X.; Baroni, S. Advanced capabilities for materials modelling with Quantum ESPRESSO. J. Phys. Condens. Matter. 2017, 29, 465901.

(30) Supka, A. R.; Lyons, T. E.; Liyanage, L.; D’Amico, P.; Al Orabi, R. A. R.; Mahatara, S.; Gopal, P.; Toher, C.; Ceresoli, D.; Calzolari, A.; Curtarolo, S.; Buongiorno Nardelli, M.; 
Fornari, M. AFLOW $\pi$ : A minimalist approach to high-throughput ab initio calculations including the generation of tight-binding hamiltonians. Comput. Mater. Sci. 2017, 136, $76-84$

(31) Buongiorno Nardelli, M.; Cerasoli, F. T.; Costa, M.; Curtarolo, S.; De Gennaro, R.; Fornari, M.; Liyanage, L.; Supka, A. R.; Wang, H. PAOFLOW: A utility to construct and operate on ab initio Hamiltonians from the projections of electronic wavefunctions on atomic orbital bases, including characterization of topological materials. Comput. Mater. Sci. 2018, 143, $462-472$.

(32) Agapito, L. A.; Curtarolo, S.; Buongiorno Nardelli, M. Reformulation of DFT $+U$ as a pseudohybrid Hubbard density functional for accelerated materials discovery. Phys. Rev. X 2015, 5, 011006.

(33) Gopal, P.; Fornari, M.; Curtarolo, S.; Agapito, L. A.; Liyanage, L. S. I.; Buongiorno Nardelli, M. Improved predictions of the physical properties of Zn- and Cd-based wide band-gap semiconductors: A validation of the ACBN0 functional. Phys. Rev. B 2015, 91, 245202.

(34) Perdew, J. P.; Burke, K.; Ernzerhof, M. Generalized gradient approximation made simple. Phys. Rev. Lett. 1996, 77, 3865-3868.

(35) Gopal, P.; De Gennaro, R.; Silva dos Santos Gusmao, M.; Al Orabi, R.; Wang Al Rahal, H.; Curtarolo, S.; Fornari, M.; Buongiorno Nardelli, M. Improved electronic structure and magnetic exchange interactions in transition metal oxides. J. Phys. Condens. Matter. 2017, 29, 444003.

(36) Perdew, J. P.; Ruzsinszky, A.; Csonka, G. I.; Vydrov, O. A.; Scuseria, G. E.; Constantin, L. A.; Zhou, X.; Burke, K. Restoring the density-gradient expansion for exchange in solids and surfaces. Phys. Rev. Lett. 2008, 100, 136406. 
(37) D’Amico, P.; Agapito, L.; Catellani, A.; Ruini, A.; Curtarolo, S.; Fornari, M., Buongiorno Nardelli; Calzolari, A. Accurate ab initio tight-binding Hamiltonians: effective tools for electronic transport and optical spectroscopy from first principles. Phys. Rev. $B$ 2016, 94, 165166.

(38) Gravier, L.; Fábián, A.; Rudolf, A.; Cachin, A.; Wegrowe, J.-E.; Ansermet, J.-P. Spindependent thermopower in $\mathrm{Co} / \mathrm{Cu}$ multilayer nanowires. J. Magn. Magn. Mater 2004, 271, 153-158.

(39) Zhang, Y. First-principles Debye-Callaway approach to lattice thermal conductivity. Journal of Materiomics 2016, 2, 237 - 247.

(40) Zhang, Y.; Skoug, E.; Cain, J.; Ozoliņš, V.; Morelli, D.; Wolverton, C. First-principles description of anomalously low lattice thermal conductivity in thermoelectric $\mathrm{Cu}-\mathrm{Sb}-\mathrm{Se}$ ternary semiconductors. Phys. Rev. B 2012, 85, 054306.

(41) Broido, D. A.; Ward, A.; Mingo, N. Lattice thermal conductivity of silicon from empirical interatomic potentials. Phys. Rev. B 2005, 72, 014308.

(42) Morelli, D. T.; Heremans, J. P.; Slack, G. A. Estimation of the isotope effect on the lattice thermal conductivity of group IV and group III-V semiconductors. Phys. Rev. B 2002, 66, 195304.

(43) Morelli, D. T.; Jovovic, V.; Heremans, J. P. Intrinsically Minimal Thermal Conductivity in Cubic I-V-VI 2 Semiconductors. Phys. Rev. Lett. 2008, 101, 035901.

(44) Szymanski, J. T. The crystal structure of Mawsonite $\mathrm{Cu}_{6} \mathrm{Fe}_{2} \mathrm{SnS}_{8}$. Can. Mineral. 1976, $14,529-535$.

(45) http://rruff.geo.arizona.edu/AMS/amcsd.php,.

(46) Imai, H.; Lee, M. S.; Iida, K.; Fujiki, Y.; Takenouchi, S. Geologic structure and mineralization of the xenothermal vein-type deposits in Japan. Econ. Geol. 1975, 70, 647-676. 
(47) Baláž, P.; Hegedüs, M.; Reece, M.; Zhang, R.-Z.; Su, T.; Škorvánek, I.; Briančin, J.; Baláž, M.; Mihálik, M.; Tešínsky, M., et al. Mechanochemistry for thermoelectrics: nanobulk $\mathrm{Cu}_{6} \mathrm{Fe}_{2} \mathrm{SnS}_{8} / \mathrm{Cu}_{2} \mathrm{FeSnS}_{4}$ composite synthesized in an industrial mill. J. Electron. Mater. 2019, 48, 1846-1856.

(48) Liu, M.-L.; Chen, I.-W.; Huang, F.-Q.; Chen, L.-D. Improved thermoelectric properties of $\mathrm{Cu}$-doped quaternary chalcogenides of $\mathrm{Cu}_{2} \mathrm{CdSnSe}_{4}$. Adv. Mater. 2009, 21, 38083812.

(49) Suekuni, K.; Tsuruta, K.; Ariga, T.; Koyano, M. Thermoelectric properties of mineral tetrahedrites $\mathrm{Cu}_{10} \operatorname{Tr}_{2} \mathrm{Sb}_{4} \mathrm{~S}_{13}$ with low thermal conductivity. Appl. Phys. Express 2012, $5,051201$.

(50) Yamanaka, T.; Kato, A. Mossbauer effect study of ${ }^{57} \mathrm{Fe}$ and ${ }^{119} \mathrm{Sn}$ in stannite, stannoidite, and mawsonite. Am. Mineral. 1976, 61, 260-265.

(51) Xi, L.; Zhang, Y. B.; Shi, X. Y.; Yang, J.; Shi, X.; Chen, L. D.; Zhang, W.; Yang, J.; Singh, D. J. Chemical bonding, conductive network, and thermoelectric performance of the ternary semiconductors $\mathrm{Cu}_{2} \mathrm{Sn}_{3}(X=$ Se, S) from first principles. Phys. Rev. B 2012, 86, 155201.

(52) Takaki, H.; Kobayashi, K.; Shimono, M.; Kobayashi, N.; Hirose, K.; Tsujii, N.; Mori, T. First-principles calculations of Seebeck coefficients in a magnetic semiconductor $\mathrm{CuFeS}_{2}$. Appl. Phys. Lett. 2017, 110, 072107.

(53) Tsujii, N.; Mori, T. High thermoelectric power factor in a carrier-doped magnetic semiconductor $\mathrm{CuFeS}_{2}$. Appl. Phys. Express 2013, 6, 043001.

(54) Conejeros, S.; Alemany, P.; Llunell, M.; Moreira, I. d. P. R.; Sánchez, V.; Llanos, J. Electronic structure and magnetic properties of $\mathrm{CuFeS}_{2}$. Inorg. Chem. 2015, 54, 48404849. 
2

3

4

5

6

7

8

9

10

11

(55) Duan, B.; Yang, J.; Salvador, J. R.; He, Y.; Zhao, B.; Wang, S.; Wei, P.; Ohuchi, F. S.; Zhang, W.; Hermann, R. P.; Gourdon, O.; Mao, S. X.; Cheng, Y.; Wang, C.; Liu, J.;

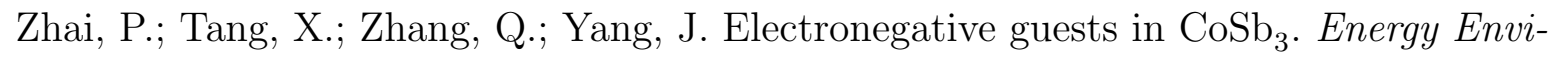
ron. Sci. 2016, 9, 2090-2098.

(56) Zhao, L. D.; Lo, S. H.; Zhang, Y.; Sun, H.; Tan, G.; Uher, C.; Wolverton, C.; Dravid, V. P.; Kanatzidis, M. G. Ultralow thermal conductivity and high thermoelectric figure of merit in SnSe crystals. Nature 2014, 508, 373. 


\section{Graphical TOC Entry}

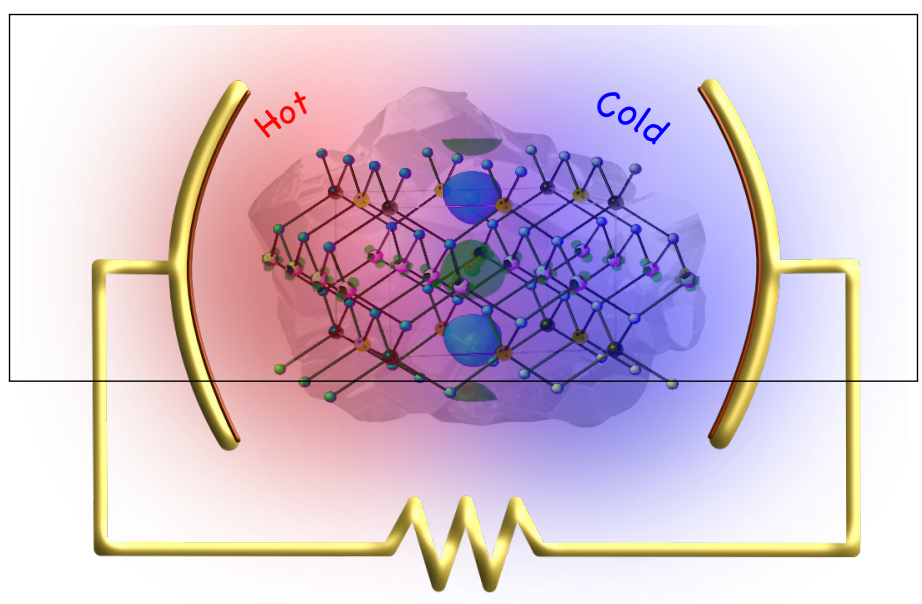

